Edited by Kiriakos Xenitidis and
Colin Campbell
Contents
- Is alcohol consumption irrelevant to outcome
in anxiety and depression?
- Depression in the workplace:
what is depression?
- Severe mental illness in prisoners worldwide

\section{Is alcohol consumption irrelevant to outcome in anxiety and depression?}

Boschloo and colleagues ${ }^{1}$ report that DSM-IV alcohol abuse is not a risk factor for unfavourable outcome in depression and anxiety disorders. This finding, which ostensibly conflicts with clinical experience, could easily be misunderstood to imply that nondependent alcohol use is irrelevant in these disorders. We do not believe this is a true finding of the study. The paper addresses DSM-IV alcohol use disorder diagnosis at baseline as a risk factor for poor outcome. It does not address continuing alcohol abuse and its effects on depression and anxiety. Latest alcohol consumption statistics show that a substantial proportion of the population drink alcohol heavily. In England, the latest estimates are that $26 \%$ of men and $17 \%$ of women over the age of 16 drink at levels that are hazardous or harmful to health. ${ }^{2}$ Clearly, only a small proportion of these receive a diagnosis of alcohol use disorder and there is no reason to believe that the situation in The Netherlands is radically different. It would be expected that a significant proportion of the sample with no alcohol use disorder diagnosis would be drinking alcohol at hazardous or harmful levels. Alcohol use disorder is therefore a poor marker for alcohol consumption. Alcohol use disorder diagnosis cannot have an effect on the course of depressive or anxiety disorders as it is a theoretical construct. It is alcohol consumption that has an effect. The current data are extracted from a larger study with different aims ${ }^{3,4}$ and contain a number of flaws when used to examine the effects of alcohol on depression and anxiety. There are no indications of levels of alcohol consumption other than alcohol use disorder diagnosis and there are no data on alcohol diagnosis at follow-up. There is no information about what treatments the sample patients received, although this is likely to have included advice to reduce alcohol consumption. Unsurprisingly, the study demonstrates that those with the poorest depression and anxiety outcomes are those with a baseline diagnosis of alcohol dependence, with a stronger effect with increasing severity of disorder. That is, those patients least likely to have reduced their alcohol consumption during the study had the poorest outcomes. There is a puzzling finding in relation to severity of alcohol diagnosis: there are more patients in the sample with the more severe diagnosis of alcohol dependence than with the less severe diagnosis of alcohol abuse. The diagnostic criteria for alcohol abuse are concerned largely with social dysfunction, whereas those for alcohol dependence are concerned with physical dependence and social dysfunction. It may be that the gap between the onset of moderate social dysfunction and the onset of physical dependence or severe social dysfunction (as determined by the diagnostic criteria) represents a small part of the deterioration resulting from sustained heavy drinking, and therefore is a poor indicator of population harmful drinking. The paper does convincingly suggest that severe alcohol dependence at baseline is a risk factor for poor outcome in anxiety and depression. It does not demonstrate that continued drinking in other groups is irrelevant to outcome.

1 Boschloo L, Vogelzangs N, van den Brink W, Smit JH, Veltman DJ, Beekman ATF, et al. Alcohol use disorders and the course of depressive and anxiety disorders. Br J Psychiatry 2012; 200: 476-84.

2 Health and Social Care Information Centre. Statistics on Alcohol: England, 2012. Health and Social Care Information Centre, 2012.

3 Penninx BWJH, Beekman ATF, Smit JH, Zitman FG, Nolen WA, Spinhoven P, et al. The Netherlands Study of Depression and Anxiety (NESDA): rationale objectives and methods. Int J Methods Psychiatr Res 2008; 17: 121-40.

4 Van Der Veen WJ, Van Der Meer K, Penninx BW. Screening for depression and anxiety: correlates of non-response and cohort attrition in the Netherlands Study of Depression and Anxiety (NESDA). Int J Methods Psychiatr Res 2009; 18: 229-39.

John Bailey, Centre for Mental Health and Society, Bangor University, UK. Email j.bailey@bangor.ac.uk; Rob Poole, Centre for Mental Health and Society, Bangor University; Susan Ruben, Betsi Cadwaladr University NHS Trust, North Wales; Catherine A. Robinson, Centre for Mental Health and Society, Bangor University, UK

doi: 10.1192/bjp.201.4.326

Authors' reply: We appreciate Bailey et al's interest in our study $^{1}$ and respond to their comments regarding the found differential effects of alcohol dependence $v$. alcohol abuse and alcohol consumption.

It is evident that heavy alcohol consumption has a major impact on public health because of its negative consequences on the onset and course of various diseases. ${ }^{2}$ However, this does not necessarily imply that the level of alcohol consumption is also causally related to the onset and persistence of psychopathology such as depression and anxiety. For example, Haynes $\mathrm{et} \mathrm{al}^{3}$ showed that heavy alcohol consumption was not a risk factor for the onset of depression and anxiety in a national sample of adults in Great Britain. Furthermore, additional analyses in our sample showed that neither heavy alcohol consumption at baseline (odds ratio $(\mathrm{OR})=0.98,95 \%$ CI $0.71-1.36)$ nor at 2-year follow-up $(\mathrm{OR}=0.98,95 \%$ CI $0.69-1.41)$ predicted the persistence of depression and anxiety.

In contrast, DSM-IV alcohol dependence has strong links with depression and anxiety. For example, previous epidemiological studies have demonstrated robust cross-sectional associations ${ }^{4,5}$ and, in addition, our study showed that alcohol dependence at baseline was an important risk factor for the persistence of depression and anxiety during 2-year follow-up $(\mathrm{OR}=1.69,95 \%$ CI 1.04-2.75, adjusted for basic covariates). ${ }^{1}$ Additional analyses further showed that this association remained significant after controlling for the possible effects of alcohol consumption $(\mathrm{OR}=2.05,95 \%$ CI 1.19-3.53, additionally adjusted for alcohol consumption). This indicates that impairments related to alcohol dependence, rather than consumption per se, have an effect on the course of depression and anxiety. Support for this hypothesis was also provided by our finding that persistence rates of depression and anxiety increased with the severity of alcohol dependence (i.e. number of alcohol dependence criteria). ${ }^{1}$

Previous studies have raised the question whether DSM-IV alcohol abuse should be considered a genuine psychiatric disorder as it was characterised by low severity in the general population and showed limited reliability and validity (see Boschloo et al ${ }^{1}$ for a discussion on this topic). This notion received further support by our finding that $95.7 \%$ of patients with alcohol abuse met only one of four abuse criteria. ${ }^{1}$ Additional analyses showed 